\title{
Maximum Entropy Production Principle and Morphological Selection in Hydrodynamic Systems ${ }^{+}$
}

\author{
Anna Birzina 1,** and Leonid Martyushev 1,2 \\ 1 Ural Federal University, Technical Physics Department, 19 Mira Str., 620002 Ekaterinburg, Russia; \\ leonidmartyushev@gmail.com \\ 2 Institute of Industrial Ecology, 20 S. Kovalevskoy Str., 620219 Ekaterinburg, Russia \\ * Correspondence: birzina.anna@gmail.com; Tel.: +7-922-189-0425 \\ + Presented at the 4th International Electronic Conference on Entropy and Its Applications, \\ 21 November-1 December 2017; Available online: http://sciforum.net/conference/ecea-4.
}

Published: 20 November 2017

\begin{abstract}
In recent decades an idea has emerged that the maximum entropy production principle can be used to select from the different regimes of development of nonequilibrium systems. According to this principle the process with maximum entropy production is most preferred among the possible non-equilibrium processes. As a consequence, entropy production can be used to find an actually observed pattern formation among the hypothetical ones. A hypothesis is introduced that entropy production can be used to find a boundary (binodal) dividing the region of absolutely stable growth from the region of growth which is unstable (metastable) with regard to arbitrary amplitude distortions. This principle has been successfully applied to analyze the interfacial morphological stability during crystallization. The objective of this study is an application of described principle in two cases. The first problem is the stability analysis of the displacement front of two fluids in the radial Hele-Shaw cell. Together with linear stability analysis (which describes the stability to infinitesimal distortions, i.e., gives the spinodal) entropy production approach allows to determinate the region of different interface forms coexistence. These boundaries are analyzed depending on the cell size, the injected flow rate, and the ratio of the fluid viscosities. The second problem is the stability of a spherical surface of a vapor bubble growing under inertia control. In this case we obtain that the entropy production in the vicinity of the bubble's distorted surface is always greater than that of the undistorted surface. Such a result indicates that the morphological phase with a distorted surface is more preferable and consequently should be observable in a real system where arbitrary perturbations occur. This allows explaining the experimentally observed roughness of the bubble surface during explosive vaporization.
\end{abstract}

Keywords: inertia controlled growth; vapor bubble growth; entropy production; morphological stability

\section{Introduction}

There is an enormous amount of theoretical studies dedicated to the evolution of two phase interfaces. Particularly, a lot of works are focused on morphological transitions from one shape of an interface between moving fluids to another. The important problem is a prediction of the size (or growth time) of an interface after which a transition takes place. Morphological transitions are traditionally studied in the frameworks of linear stability analysis. A harmonic perturbation is introduced into a model and is analyzed in linear approximation. If the perturbation increases with time, the interface is unstable and morphological transition occurs. The interface stability with regard to infinitesimal perturbations can only be judged from such calculations. Consequently, linear 
analysis has one essential drawback: it cannot answer the question when the interface will become morphologically unstable if perturbation is not infinitely small. But this question is very important in practice. Indeed, it is difficult to expect only infinitely small perturbations in a real experiment.

There is an idea that the maximum entropy production principle (MEPP) can be used to describe the evolution of nonequilibrium systems and morphological transitions [1-3]. The method based on MEPP was successfully used earlier for analysis of morphological transitions during nonequilibrium crystallization [4]. The entropy production was calculated for distorted and undistorted evolving crystal phase interface and the process with the largest entropy production is assumed to be nonlinearly stable. On the basis of these results we proposed that a size of stability of the fluid interface with regard to arbitrary perturbations can be determined by comparing entropy productions in a unit volume near distorted and undistorted moving interfaces between two fluids. We say that the role of entropy production for the considered morphological transitions is similar to the role of chemical potential for equilibrium phase transitions, i.e., entropy production allows to determine the boundary of the region of the absolutely stable interface growth. In other words, we can find a bimodal of morphological transition. And in its turn the linear approximation allows to determine a spinodal of morphological transition, i.e., a boundary of the absolutely unstable region. The region between these two boundaries may be called the metastable area where perturbation can either disappear or grow depending on perturbation amplitude.

In this work the results of morphological stability analysis for two problems will be discussed. The first one is the problem of interfacial stability of two fluids with one fluid displacing the other in a Hele-Shaw cell. The second problem is the analysis of the morphological stability of the growing vapor bubble interface.

\section{Morphological Selection during Fluid Displacement in the Radial Hele-Shaw Cell}

We considered a slow quasi-stationary displacement of a fluid by another less viscous one with constant flow rate $Q$. Both fluids assumed to be immiscible and incompressible. The Hele-Shaw cell consists of two plane parallel circular glasses spaced by small (compared with radial cell size) distance $b$. During the displacement the initially circular interface may become unstable and so-called viscous fingers may appear on the interface. The perturbation of the interface is represented in the form $r=R+\delta_{0} \cos (n \varphi)$, where $R$ is the radius of the circular interface, $\delta_{0}$ is the amplitude of perturbation, $n$ is the mode of perturbation, and $r$ and $\varphi$ are the polar coordinates.

From the linear analysis [5] we can find that the critical size $R s$ when the fluid interface becomes morphologically unstable with respect to infinitesimal perturbations (the spinodal) is determined from the following equation:

$$
1+n F=0
$$

Here

$$
F=\left(\frac{M_{2}}{M_{1}}-1\right)\left(1+\left[\left(n^{2}-1\right) \frac{\beta}{R}-\alpha \gamma\left(\frac{Q}{2 \pi R}\right)^{\gamma}\right]\right)
$$

$$
\times \frac{2 \pi}{Q} \frac{M_{1} M_{2}}{M_{2}-M_{1}}\left[\frac{M_{2}}{M_{1}} \frac{1+\left(R_{0} / R\right)^{2 n}}{1-\left(R_{0} / R\right)^{2 n}}+\frac{1-\left(R / R_{\infty}\right)^{2 n}}{1+\left(R / R_{\infty}\right)^{2 n}}+n \alpha \gamma\left(\frac{Q}{2 \pi R}\right)^{\gamma} \frac{2 \pi M_{2}}{Q}\right]
$$

where $M_{i}=b^{2} / 12 \mu_{i}, R_{0}$ is the radius of the hole through which the displacing fluid is injected, $R_{\infty}$ is the size of the Hele-Shaw cell, $\mu_{i}$ is the fluid viscosity ( $i=1$ and 2 for the displacing and the displaced fluid respectively), $\alpha, \beta$ and $\gamma$ are some parameters (see [5] for details).

The analysis of change of entropy production in the vicinity of the fluid interface gives that the bimodal of the morphological transition can be determined from the solution of the Equation [6]:

$$
1+2 n F=0
$$

These two equation have only numerical solutions and their analysis may be found in [6]. In brief, we can say the following. The binodal and the spinodal exhibit similar behaviors, both become larger with cell size $R_{\infty}$ increase and smaller with displacement rate $Q$ growth. Also their values 
depend on the fluid viscosity ratio. The binodal is always smaller than the spinodal for one and the same harmonic $n$. However, spinodals and binodals relating to different disturbing harmonics can mutually intersect. The higher the viscosity of the displacing fluid, the more diverse and complicated the sequence of the morphological transitions from the circular to the cosine-like interfaces becomes (see Figure 1).

The theoretical values of the binodal and spinodal were quantitatively confirmed for small viscosity ratio (the displacement of silicon oil by air was studied) [7].

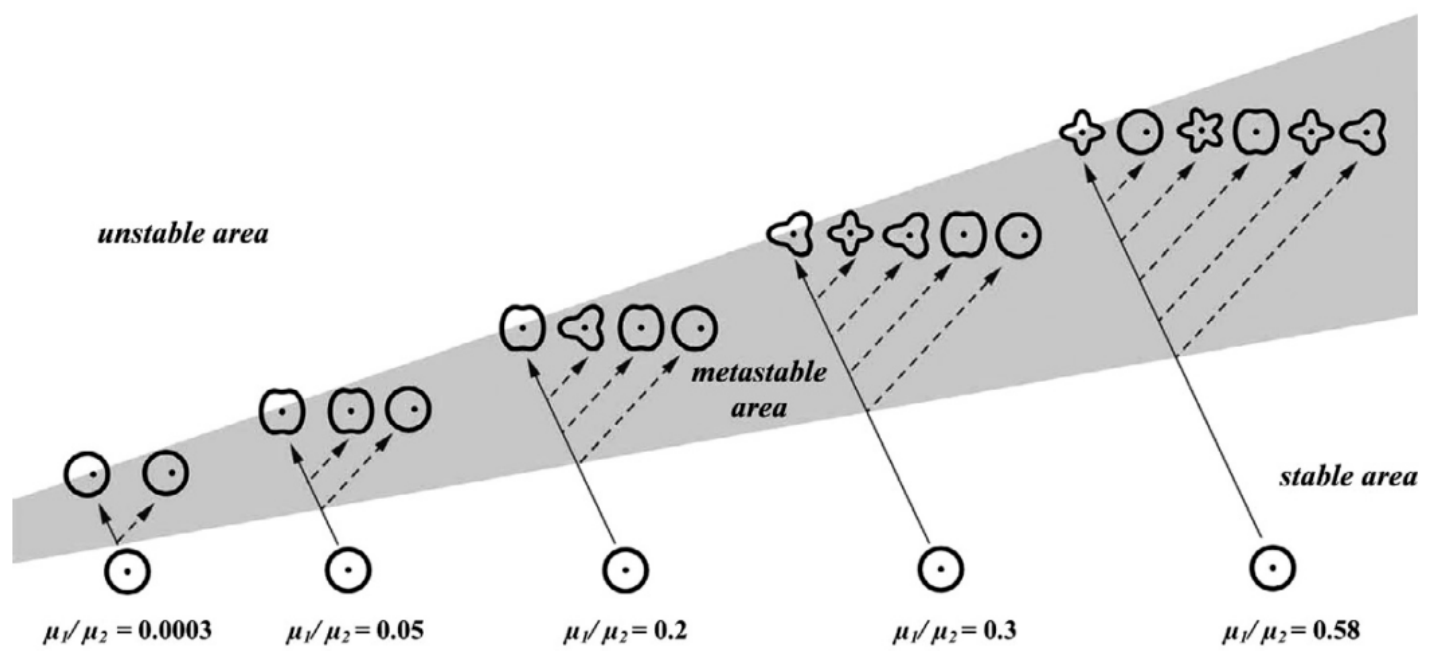

Figure 1. The possible sequence of structures during the displacement in the Hele-Shaw cell at different viscosity ratios $\mu_{1} / \mu_{2}$ [6].

\section{The Morphological Stability of a Vapor Bubble during Inertia-Controlled Growth}

Another problem under consideration is the stability analysis of the vapor bubble surface growing under inertia control. In this approximation, the bubble growth is fully determined by pressure difference between the interior and the exterior of the bubble, whereas heat processes related to liquid cooling caused by vaporization are neglected.

The mathematical formulation of the problem is as follows. A spherical vapor bubble in a superheated liquid are considered. The liquid density is considerably higher than the vapor density. The fluids under consideration are regarded as incompressible and their viscosities are neglected. The pressure inside the bubble is assumed constant, the pressure in the liquid at a large distance from the bubble also deemed constant.

The discussion of the morphological stability of a spherical interface between two phases with regard to the infinitesimal interfacial distortions can be found in the classical papers [8,9]. Following this traditional method, in dimensionless variables (normalized to the critical nucleation radius) the phase interface can be written as $r_{s}=R+\delta Y_{n}$, where $R$ is the radius of undistorted bubble surface, $Y_{n}$ is a spherical harmonic of degree (mode) $n$ and $\delta$ is the distortion amplitude $(\delta<R)$. Considering the bubble size much greater than the critical nucleation radius $(R \gg 1)$ and assuming that, at some moment of time when the bubble reaches the size $R_{0}$, there is a distortion with the amplitude $\delta_{0}$ and the zero rate on the bubble's surface $\left(\delta\left(R_{0}\right)=\delta_{0}, d \delta / d R\left(R_{0}\right)=0\right)$ in the first order in $\delta$ it is possible to obtain the following approximate equation for changing the amplitude of the distortion with bubble size $R$ :

$$
\frac{d \delta}{d R}=-\frac{2 \delta_{0}}{8 N R_{0}+1} \frac{R_{0}}{R^{2}}\left(1-\frac{R_{0}}{R}\right)
$$

where $N=[3(n-1)(n+1)(n+2)]^{-1}$.

It follows from the last equation that distortion amplitude decreases with the growth of a vapor bubble for any bubble size $R>R_{0} \gg 1$. Therefore, in the assumed approximations, the distortion 
always decays and such decay occurs independently of the parameters of a liquid and the degree of non-equilibrium of a system, where this bubble grows. Thus, the linear analysis of morphological stability cannot explain the unstable behavior of the interface under inertial growth as observed in the experiments [10-12].

Now let us consider the morphological stability of the bubble surface from the standpoint of the MEPP. According to the methods of irreversible thermodynamics, it can be shown [13] that the difference of entropy productions $\Delta \Sigma$ of the elementary volumes of the bubble in the vicinity of the disturbed and undisturbed surface can be written for the system under consideration as

$$
\Delta \Sigma \propto R \frac{d \delta}{d R}+\delta=\frac{\delta_{0}}{8 M R_{0}+1}\left(\frac{R_{0}^{2}}{R^{2}}+8 M R_{0}\right)
$$

It is follows from this relation that the entropy production in the vicinity of the bubble's distorted surface is always greater than that of the undistorted surface $(\Delta \Sigma>0)$. According to MEPP such a result indicates that the morphological phase with a distorted surface is more preferable from the thermodynamic perspective and, consequently, should be observable in a real system where arbitrary perturbations occur. This theoretical result makes it possible to give for the first time the qualitative explanation of the surface instability (roughness) observed in the experiments [10-12]. Previously, such observations could only be theoretically explained using considerable complications in the mathematical model of bubble growth.

\section{Conclusions}

It was shown that thermodynamic approach based on the maximum entropy production principle can be used for analysis of morphological stability of interfaces in two fluid phase systems. The considered method is more favorable rather than using only the traditional linear analysis because the experimental validation of theoretical results is possible.

Acknowledgments: The reported study was funded by RFBR according to the research project No. 16-31-00255 мол_a and scientific project No. 1.4539.2017/8.9.

Author Contributions: All authors made substantial contributions to the conception, calculation, and writing of this manuscript. All authors have read and approved the final version of the manuscript.

Conflicts of Interest: The authors declare no conflict of interest.

\section{References}

1. Ben-Jacob, E.; Garik, P. The formation of pattern in non-equilibrium growth. Nature 1990, 343, 523-530, doi:10.1038/343523a0.

2. Ozawa, H.; Ohmura, A.; Lorenz, R.D.; Pujol, T. The second law of thermodynamics and the global climate system: A review of the maximum entropy production principle. Rev. Geophys. 2003, 41, doi:10.1029/2002RG000113.

3. Hill, A. Entropy production as the selection rule between different growth morphologies. Nature 1990, 348, 426-428, doi:10.1038/348426a0.

4. Martyushev, L.M. Entropy Production and Morphological Selection in Crystal Growth. In Beyond the Second Law. Entropy Production and Non-Equilibrium Systems; Dewar, R.C., Lineweaver, C.H., Niven, R.K., Regenauer-Leib, K., Eds.; Springer: Berlin/Heidelberg, Germany, 2014; pp. 383-396.

5. Martyushev, L.M.; Birzina, A.I. Specific features of the loss of stability during radial displacement of fluid in the Hele-Shaw cell. J. Phys. Condens. Matter 2008, 20, doi:10.1088/0953-8984/20/04/045201.

6. Martyushev, L.M.; Birzina, A.I. Entropy production and stability during radial displacement of fluid in Hele-Shaw cell. J. Phys. Condens. Matter 2008, 20, doi:10.1088/0953-8984/20/46/465102.

7. Martyushev, L.M.; Birzina, A.I. Metastability at the Displacement of a Fluid in a Hele-Shaw Cell. JETP Lett. 2014, 99, 446-451, doi:10.1134/S0021364014080104.

8. Plesset, M.S. On the stability of fluid flows with spherical symmetry. J. Appl. Phys. 1954, 25, doi:10.1063/1.1721529. 
9. Plesset, M.S.; Mitchell, T.P. On the stability of the spherical shape of a vapor cavity in a liquid. Q. Appl. Math. 1956, 12, 419-430.

10. Shepherd, J.E.; Sturtevant, B. Rapid evaporation at the superheat limit. J. Fluid Mech. 1982, 121, doi:10.1017/S0022112082001955.

11. Frost, D.; Sturtevant, B. Effects of ambient pressure on the instability of a liquid boiling explosively at the superheat limit. J. Heat Transf. 1986, 108, doi:10.1115/1.3246940.

12. Lee, H.S.; Merte, H.J. The origin of the dynamic growth of vapor bubbles related to vapor explosions. J. Heat Transf. 1998, 120, doi:10.1115/1.2830041.

13. Martyushev, L.M.; Birzina, A.I.; Soboleva, A.S. On the morphological instability of a bubble during inertiacontrolled growth. Physica A 2018, under review.

(C) 2018 by the authors. Licensee MDPI, Basel, Switzerland. This article is an open access article distributed under the terms and conditions of the Creative Commons Attribution (CC BY) license (http://creativecommons.org/licenses/by/4.0/). 